\title{
Analysis of Pupils’ Difficulties in Solving Questions Related to Fractions: The Case of Primary School Leaving Examination in Tanzania
}

\author{
Joyce Lazaro Ndalichako \\ National Examinations Council of Tanzania, Dar es Salaam, Tanzania \\ Email: jndalichako@yahoo.com \\ Received July 2013
}

\begin{abstract}
In this paper, analysis of the performance of candidates in Mathematics in Primary School Leaving Examination was conducted with the aim of highlighting difficulties encountered in solving fraction-related problems. The analysis has indicated that a considerable number of candidates could not perform correct operations related to fractions. They tended to confuse fraction concepts with whole number concepts. For instance, in questions involving addition of fractions, they were treating numerators and denominators as separate entities. Possible reasons for such difficulties in solving questions related to fractions include lack of understanding of appropriate procedures to apply in solving a problem, the complexity of the task, over-generalization of procedures even in situations which are inappropriate. It is recommended that a protocol analysis be conducted in order to gain a deep understanding of the thought process of candidates when attempting questions related to fractions so that teachers may use relevant teaching methods that would facilitate meaningful learning of fractions.
\end{abstract}

Keywords: Distracter Analysis; Fraction Computations; Misconceptions

\section{Introduction}

Educators and researchers agree that most students encounter major problems in learning fractions (Buzek \& Bieck, 1993; Newstead \& Murray, 1998; Pitkethly \& Hunting, 1996; Tzur, 1999). It is well documented that fractions are among the most complex concepts that children encounter in their years of primary education (Saxe, Taylor, McIntosh, \& Geahart, 2005; Stafylidous \& Vosniodou, 2004; Idris \& Narayanan, 2011). It has also been asserted that learning fractions is probably one of the most serious obstacles to the mathematical maturation of children (Behr, Harel, Post, \& Lesh, 1993). Different views are expressed as factors contributing to students' difficulties in learning fractions. Possible reasons stated for such difficulties include the fact that there are many rules associated with the computation of fractions which are more complex than those of natural numbers. Moss and Case (1999) established that students difficulties in learning fractions emanate from the fact that most teachers devote too much time to teaching the procedures of manipulating fractions and too little time to teaching their conceptual meaning. Often students' competence with a rote procedure acts as a major obstacle for their conceptual understanding of fractions which in turn makes them unable to monitor their work. They can only check their answers by repeating the rote procedure used and fail to judge the reasonableness of their answer because they are too confident with their approaches.

In this paper, analysis of the general performance of candidates in Mathematics in Primary School Leaving Examination was conducted with the aim of highlighting difficulties encountered by pupils in solving mathematical problems. More specifically questions related to fractions have been given due attention because review of items in Mathematics examination indicates that candidates did not perform well in questions from this area.

\section{The System of Education in Tanzania}

The system of education in Tanzania is pyramidal in nature ranging from Primary to University level. The structure of formal education is 2-7-4-2-3 comprising of 2-years of pre-primary education, 4 years of secondary ordinary education level; 2 years of secondary advanced level and a minimum of 3 years of University education. Progression from one level to another depends on the performance in the summative examination specifically set at each respective level of education. Since the focus of this paper is on primary school leaving examination, the following sections highlight the structure of the education at Primary level and the nature of its final examination.

\section{The Structure of Primary Education in Tanzania}

Primary education is a seven-year cycle which is compulsory in enrolment and attendance for children aged 7 years. The cycle is divided into "Standards" representing the respective year of study (i.e. Standard I to VII) for pupils. In this paper the term Grade will be used to imply Standard. Promotion from one Grade to the next is automatic. Repetition of a class is only allowed in Grade I-IV. For Grades V-VII, repetition can only be allowed if one has approval from the relevant authorities based on genuine reasons such as illness. Grade VII marks the end of Primary education and all pupils are subjected to Primary School Leaving Examination which is used for selection of those who qualify to go on with secondary education. Those who are not progressing 
to secondary education can join vocational training or pursue any other options leading into the world of work.

There are six major subject areas that are taught at Primary levels namely Languages (Kiswahili, English and French); Social Studies (History, Geography, and Civics); Science and Technology (Science and ICT); Life Skills (Vocational Studies and Personality and Sports); Ethics and Religious studies; and Mathematics (Tanzania Institute of Education, 2004). English and Kiswahili Languages as well as Mathematics are regarded as fundamental subjects and are given greater weight in terms of hours of teaching than other subjects. The duration of a period is $30 \mathrm{mi}-$ nutes for Grade I-II and 40 minutes for Grade III-VII. Table 1 indicates the number of periods per subjects taught in Primary Schools.

\section{The Primary School Leaving Examination}

The Primary School Leaving Examination (PSLE) marks the completion of primary education cycle. The PSLE consists of five papers assessing seven core subjects in primary education curriculum. The papers include Mathematics, English Language, Kiswahili, Science and Social Studies (Civics, Geography, and History). The PSLE is set by the National Examinations Council of Tanzania (NECTA) and administered in schools through a close supervision of the regional and district authorities. Each paper consists of 50 multiple choice items with 5-options where candidates are supposed to select the correct answer.

Development of items for PSLE is done carefully by ensuring that all questions are set within the respective subject syllabus and conform to the key principles in setting examination items. Experienced primary school teachers are invited by NECTA to submit examination items in accordance with the guidelines given. The items submitted are subjected to moderation by

Table 1.

Subject taught and number of periods per week.

\begin{tabular}{cccc}
\hline \multirow{2}{*}{ S/N } & Subject & \multicolumn{2}{c}{ Number of Periods per Week per Grade } \\
\cline { 3 - 4 } 1. & Kiswahili & 6 & III - VII \\
\hline 2. & English & 7 & 7 \\
3. & Mathematics & 7 & 7 \\
4. & Science & 2 & 7 \\
5. & Geography & - & 4 \\
6. & History & - & 3 \\
7. & Civics & - & 2 \\
8. & Vocational Skills & 3 & 2 \\
9. & Personality and Sports & 2 & 2 \\
10. & ICT & 1 & 2 \\
11. & Religious Studies & 2 & 2 \\
12. & French & 2 & 2 \\
& Total & $\mathbf{3 2}$ & $\mathbf{4 2}$ \\
\hline
\end{tabular}

Note: Source: Tanzania Institute of Education, (2004). Primary Education Curriculum. subject officers and other education experts such as inspectors and curriculum developers. In moderation of items, clarity of the items, difficulty level and content coverage are among the areas covered. Furthermore, all distracters are reviewed to ensure that they are equally attractive. Misconceptions and possible errors that can be made by pupils are used in developing distracters that are likely to attract pupils who have partial knowledge in the respective area.

\section{Performance of Candidates in PSLE 2012}

A total of 894,839 candidates registered for PSLE in 2012. The number of candidates who sat for the examination was 865,827 which is equivalent to 96.76 percent of registered candidates. The overall percentage of candidates who passed the examination was 30.72 . When the performance is disaggregated subject wise, Mathematics can be seen as one of the subjects which contributed significantly in pulling down the overall performance of candidates as only 18.74 percent of the candidates passed Mathematics examination. Table 2 indicates the performance of the candidates in PSLE 2012.

It can be seen from Table 2 that when compared to other subjects, performance in mathematics is quite low. The item analysis data for the subject revealed that item difficulty for mathematics ranged from .10 to .75 while the difficulty level fraction items ranged from .10 to .45 indicating that fraction was among the most poorly performed topics in the examination (NECTA, 2013).

\section{Overview of Challenges in Learning Fractions}

Fractions play a central role in mathematics learning. They are theoretically important because they build a foundation which helps the pupils to successfully learn topics related to percentage, ratios and decimal numbers. Fractions require a deeper understanding of computational procedures than that typically required with whole numbers. In Tanzania, the topic of fractions is an integral part of primary school mathematics syllabus which is introduced as early as pupils start Grade I, yet it is one of the most difficult areas for pupils to master. Siebert and Gaskin, (2006) contended that children are bound to find fractions computations arbitrary, confusing and easy to mix up unless they receive assistance in understanding what fractions and fraction operations mean. For instance when fraction addition and subtraction problems have the same denominator the denominator is maintained in the answer; but that is not the case for fraction multiplication

Table 2.

Subject performance in PSLE 2012.

\begin{tabular}{llll}
\hline \multirow{2}{*}{ Subject } & Candidates Sat & \multicolumn{2}{c}{ Candidates Passed } \\
\cline { 3 - 4 } & & Number & Percent \\
\hline Kiswahili & 865,173 & 354,588 & 41,0 \\
English Language & 865,176 & 182,145 & 21.0 \\
Social Studies & 865,281 & 247,448 & 28.6 \\
Mathematics & 865,221 & 162,078 & 18.7 \\
Science & 865,048 & 358,731 & 41.5 \\
\hline
\end{tabular}

Source: NECTA, (2012). Primary School Leaving Examination Results Statistics. 
and division. They suggested that emphasis should be on conceptual understanding rather than procedural understanding of operations related to fractions for meaningful learning.

Gould, Outhred, and Mitchelmore (2006) in their research aimed at understanding student reasoning and misconceptions related to fractions, they noted that students perceived fractions as parts of the sets rather than parts of the whole. In the parts of a set conception of fractions $1 / 4$ can be interpreted as meaning one object out of four; synonymous to the counting activity which assumes that 1 represents a whole number and 4 represents a whole number as well. Mack (1990) observed that computational algorithms involving fractions prevent students from even trying to reason out or make sense of fraction in real life situations. For instance, the "invert-and-multiply" algorithm for dividing fractions does not develop naturally from students using manipulative (Borko et al., 1992). Students tend to not only remember the incorrect algorithms involving fractions but also have more faith in them compared to their own reasoning.

\section{Statement of the Problem}

The important role of mathematics in helping the students to develop logical thinking is well acknowledged by educators and researchers. Recognizing that role, the education system in Tanzania is also putting a lot of emphasis in mathematics from primary to secondary levels of education. With the exception of English Language, the number of periods assigned to mathematics subject starting from Grade I is higher than the number of periods in other subjects. In the Certificate for Secondary School Examination, candidates who fail in mathematics cannot be awarded first or second division even if they have excellent grades in all of the remaining subjects. The penalty exercised for failing mathematics is meant to encourage students to work hard in the subject so that in turn they establish a solid foundation to pursue other mathematics-related subjects and enhance their logical thinking. However, the performance in mathematics at both primary and secondary levels of education remains unsatisfactory for many years.

\section{Objective of the Study}

The objective of the study was to identify areas in mathematics in PSLE in which the majority of candidates were not able to obtain a correct answer and conduct analysis of the distracters in order to identify possible reasons that may have made the candidates unable to obtain a correct answer. It is expected that the analysis of items will assist educators and policy makers in realizing the difficulties encountered by candidates in attempting to solve mathematical problems and thereby devising better approaches in training mathematics teachers so that they can be able to address such difficulties in teaching and learning process. It is also anticipated that the findings will help to fill gaps in research in this area and stimulate further studies.

\section{Analysis of Candidates’ Responses to Fraction Items}

In this section the analysis of candidates responses to items related to fractions is presented. The fraction items ranged from simple operations of adding and subtracting fractions to complex ones involving finding the square roots of fractions. For each item, the distracters that were selected by many candidates were analyzed with the aim of identifying possible sources of errors and difficulties encountered by students. The analysis of specific fraction questions is hereby presented:

Question 7:

$$
5 \frac{3}{5}-1 \frac{2}{3}=\text { A. } 4 \frac{1}{15} \text { B. } 4 \frac{1}{2} \text { C. } 3 \frac{1}{15} \text { D. } 4 \frac{14}{15} \text { E. } 3 \frac{14}{15}
$$

Question 7 was about subtracting mixed fractions. A total of 860,028 candidates attempted this question where only 283,399 (32.76\%) were able to select the correct answer E. Distracter A $3 \frac{14}{15}$ was more attractive than others as 177,216 (20.49\%) selected it (NECTA, 2013). These candidates used an incorrect procedure for subtracting mixed fractions with different denominators. They treated whole numbers in isolation of their related fractions. They are likely to have selected that option through an incorrect computation approach as follows:

$$
5 \frac{3}{5}-1 \frac{2}{3}=(5-1)\left(\frac{3}{5}-\frac{2}{3}\right)=4\left(\frac{9-10}{15}\right)=4\left(\frac{1}{15}\right)
$$

The candidates who selected A $4 \frac{1}{15}$ are likely to have used the same incorrect procedure to arrive at their solution. Although they obtained a negative fraction, they simply ignored it and used the absolute value of the fraction. This indicates that the candidates did not have a good understanding of procedures required in subtracting mixed fractions.

On the other hand 18.04 percent of the candidates selected B $4 \frac{1}{2}$ which was not a correct answer. These candidates had a similar problem to those who selected option A as they treated the whole numbers and fractions in isolation of each other. However, their problem was compounded by the fact that they treated the numerators and denominators separately as they did not find the common denominator first. In their incorrect computation, they first subtracted the whole numbers as follows:

$$
5 \frac{3}{5}-1 \frac{2}{3}=(5-1)\left(\frac{3}{5}-\frac{2}{3}\right)=4\left(\frac{3-2}{5-3}\right)=4 \frac{1}{2}
$$

Candidates' prior learning of whole numbers and the manner in which fractions were introduced to them at early stages of learning of fractions may have affected their conceptual understanding of proper algorithms to use. Post et al. (1993) and Moss and Case (1999) have demonstrated that children's whole number schemes can interfere with their learning of fractions. It is quite possible for such a situation to have happened to the candidates who could not answer the item correctly because in Tanzania operations about fractions are introduced to them in Grade IV where they have already acquired a considerable knowledge about whole numbers and their operations. In Grades I-III children are only taught about recognition of different fractions. Question 8:

$$
1 \frac{1}{2}+3 \frac{1}{2}+\frac{3}{8}=\text { A. } 4 \frac{5}{12} \text { B. } 5 \frac{1}{4} \text { C. } 5 \frac{3}{8} \text { D. } 5 \frac{5}{8} \text { E. } 4 \frac{3}{8} \text {. }
$$

Question 8 was measuring candidates' ability to add mixed fractions. Only 37.4 percent of the candidates were able to select the correct answer which was C $5 \% 3$. Among the distracters, option A attracted more candidates than other distracters. The 22.23 percent of candidates who selected A were using an $4 \frac{5}{12}$ incorrect algorithm for adding fractions with different denominators. They simply added the numerators and denominators as they were provided and then added the whole numbers separately as follows:

$$
1 \frac{1}{2}+3 \frac{1}{2}+\frac{3}{8}=(1+3) \frac{1+1+3}{2+2+8}=4 \frac{5}{12}
$$

Such candidates had a fundamental problem of understanding the essence of fractions as they treated numerators and de- 
nominators as two different numbers that do not constitute a unique entity.

Question 14: Multiply the square roots of $1 \frac{18}{32}$ and $11 \frac{1}{9}$.

$$
\begin{array}{lllll}
\text { A. } 2 \frac{1}{2} & \text { B. } 2 \frac{1}{12} & \text { C. } 3 \frac{5}{6} & \text { D. } 11 \frac{18}{288} & \text { E. } 4 \frac{1}{6}
\end{array}
$$

Question 14 required the candidates to find the square roots of the numbers given and then multiply. This question was the most difficulty item in the whole examination with the difficulty index of .1. Only 87,465 candidates out of 848,739 who attempted it obtained a correct answer (NECTA, 2013). The majority of the candidates (397,841 equivalent to $45.99 \%$ ) chose option D which was not a correct answer. These candidates neglected the key requirement in the item of multiplying the square roots of the given mixed fractions and attempted to multiply the given fractions as they were given. Moreover, they used an incorrect algorithm for multiplying the fraction in the sense that they multiplied the whole numbers and the fractions separately:

$$
1 \frac{18}{32} \times 11 \frac{1}{9}=1 \frac{18}{32} \times 11 \frac{1}{9}=(1 \times 11) \frac{18 \times 1}{32 \times 9}=11 \frac{18}{288} .
$$

Ideally, candidates should have converted the given mixed fractions into improper fractions before they started to perform the computation as required in the question.

Question 17: Find the product of 15 and 20 .

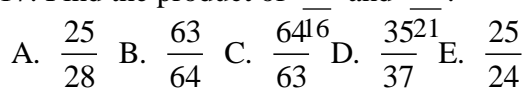

Question 17 required candidates to find the product of the given fractions. Only 28.62 percent of the candidates selected the correct answer which was A. Contrary to what was asked, the majority of candidates (30.81 percent) selected option D which was obtained by finding the sum of the fractions given instead of the product as required. They failed to distinguish the fact that the sum is obtained by adding the numbers while the product is obtained by multiplying the numbers. Their problem of not complying with the requirement of the question was compounded by the fact that even their addition procedure was incorrect. Instead of finding a common denominator first, the candidates treated the numerators and denominators as separate numbers and incorrectly added the fractions as follows:

$$
\frac{15}{16}+\frac{20}{21}=\frac{15+20}{16+21}=\frac{35}{37} \text {. }
$$

Candidates who selected this option show a lack of understanding of the conceptual basis of arithmetic procedures involving fractions. This suggests that students tend to view fractions as isolated digits, treating the numerator and denominator as separate entities that can be operated on independently. Such perception leads them to the use of incorrect algorithms.

Question 20: What number should be added to $\left(\frac{5}{2}+\frac{5}{4}\right)$ to get $\frac{1}{8}$ ?

$$
\begin{array}{lllll}
\text { A. } 3 \text { B. } 2 \frac{3}{4} & \text { C. } 3 \frac{5}{8} & \text { D. } 3 \frac{1}{2} & \text { E. }-3 \frac{5}{8}
\end{array}
$$

This item was one among the most difficulty items as only 14 percent of the candidates got the item correctly by selecting the correct answer E. On the other hand, 25.67 percent of the candidates selected C $3 \frac{5}{8}$ which was equivalent to the correct answer in absolute value but could not be the correct answer because the question required them to find a number which, if added to the sum of the two fractions given the answer would be $1 / 8$. Candidates could have used the process of estimation to realize that $3 \frac{5}{8}$ was already greater than $1 / 8$ even before adding the two given fractions.

Careful analysis of the options given for this item indicates that test-wise candidates would have been able to obtain the correct answer by elimination process because the values for all the distracters were already greater than the sum that was to be obtained. In order to get the fraction which was less than the numbers that were added, a third number must have been a negative.

\section{Conclusion}

In this paper, analysis of Primary School Leaving Examination questions related to fractions was conducted. The analysis revealed that a considerable number of candidates could not perform correcting operations related to fractions. They tended to confuse fraction concepts with whole number concepts. For instance, in questions involving addition of fractions, they were treating numerators and denominators as separate entities. Possible reasons may be attributed to pupils' difficulty in solving questions related to fractions. These include lack of understanding of appropriate procedure to apply in solving a problem and complexity of the task. Over-generalization of procedures could also pose a problem to pupils and make them apply inappropriate procedures for a given problem. For instance multiplying a number makes it bigger. However, multiplying a number with a fraction or a decimal numbers less than one makes the principle untenable and that could create a problem to students who have such generalization.

This paper was limited to multiple choice items where candidates were only selecting the correct answer among the given options. It is very likely that if the candidates were asked to construct their own answers a lot of misconceptions related to fractions would have been revealed. Therefore it is recommended that a study should be conducted in order to gain a deep understanding of the thought process of candidates when attempting questions related to fractions. Understanding of their thought process is very essential to enable teachers to use relevant teaching methods that would facilitate meaningful learning to the pupils. On the other hand, teachers teaching approaches should be investigated with the aim of identifying their professional training needs, if any, in developing conceptual understanding of fractions.

\section{REFERENCES}

Behr, M., Harel, G., Post, T., \& Lesh, R. (1992). Rational number. ratio and proportion. In D. Grouws (Ed.), Handbook of research on mathematics teaching and learning. New York: MacMillan.

Bezuk, N., \& Bieck, M. (1993). Current research on rational numbers and common fractions: Summary and implications for teachers. In D. Owens (Ed.), Research ideas for the classroom: Middle grade mathematics. NY: MacMillan.

Borko, H., Eisenhart, M., Brown, C., Underhill, R., Jones, D., \& Agard, P. (1992). Learning to teach hard mathematics: Do novice teachers and their instructors give up too easily? Journal for Research in Maths Education, 23, 194-222. http://dx.doi.org/10.2307/749118

Gould, O., \& Mitchelmore (2006). Conference proceedings of the 2006 meeting of the Mathematics education Research Group of Australia (MERGA): Australia.

Idris, N., \& Narayanan, L. M. (2011). Error patterns in addition and subtraction of fractions among form two students. Journal of Mathematics Education, 4, 35-54.

Mack, N. (1990). Learning fractions with understanding: Building on 


\section{J. L. NDALICHAKO}

informal knowledge. Journal for Research in Mathematics Education, 21, 16-32. http://dx.doi.org/10.2307/749454

Moss, J., \& Case, T. (1999). Developing children's understanding of the rational numbers: A new model and an experimental curriculum. Journal for Research in Mathematics Education, 30, 122-148. http://dx.doi.org/10.2307/749607

NECTA (2012). Primary school leaving examination results statistics: National, regional, district, and subject performance. NECTA, Dar es Salaam.

NECTA (2013). Analysis of Candidates' responses to primary school leaving examination questions for the year 2012: Mathematics. NECTA, Dar es Salaam.

Newstead, K., \& Murray, H. (1998). Young students' constructions of fractions. In A. Olivier, \& K. Newstead (Eds.), Proceedings of the 22nd Conference of the International Group for the Psychology of Mathematics Education, 3, 295-303. Stellenbosch, South Africa.

Pitkethly, A., \& Hunting, R. (1996). A review of recent research in the area of initial fraction concepts. Educational Studies in Mathematics, 30, 5-38. http://dx.doi.org/10.1007/BF00163751

Saxe, G. B., Taylor, E..V., McIntosh, C., \& Geahart, M. (2005). Representing fractions with standard notations: A development analysis. Journal for Research in Mathematics Education, 36, 137-157.

Siebert, D., \& Gaskin, N. (2006). Creating, naming and justifying fractions. Teaching Children Mathematics, 12, 394-400.

Stafylidous, S., \& Vosniodou, S. (2004). The development of students' understanding of the numerical value of fractions. Learning and Instruction, 14, 503-518.

http://dx.doi.org/10.1016/j.learninstruc.2004.06.015

Tanzania Institute of Education (2004). Primary education curriculum for Tanzania Mainland. Dar es salaam. (Unpublished Document).

Tzur, R. (1999). An integrated study of children's construction of improper fractions and the teachers role in promoting that learning, Journal for Research in Mathematics Education, 30, 390-416. http://dx.doi.org/10.2307/749707 